\title{
Productivity of L-DOPA in in vitro shoots of Mucuna pruriens var. utilis enhanced by gamma radiation
}

\author{
Detmontree Wachisunthon ${ }^{1}$, Sorrapetch Marsud ${ }^{2}$, Subhadhcha Poonsatha ${ }^{2}$, Suwimol Jetawattana ${ }^{3}$, Worapan Sitthithaworn ${ }^{1 *}$ \\ ${ }^{1}$ Faculty of Pharmacy, Srinakharinwirot University, Nakhonnayok, Thailand. \\ ${ }^{2}$ Medicinal Plant Research Institute, Department of Medical Sciences, Ministry of Public Health, Nonthaburi, Thailand. \\ ${ }^{3}$ Thailand Institute of Nuclear Technology Public Organization, Nakhonnayok, Thailand.
}

\begin{tabular}{l}
\hline ARTICLE INFO \\
\hline Received on: 06/08/2020 \\
Accepted on: $23 / 11 / 2020$ \\
Available online: 05/01/2021 \\
\\
\hline Key words: \\
L-DOPA, Mucuna pruriens \\
var. utilis, gamma radiation, \\
UPLC.
\end{tabular}

\begin{tabular}{l}
\hline ABSTRACT \\
This research aimed to study the ability to produce levodopa (L-DOPA) in an in vitro shoot culture of Mucuna pruriens \\
var. utilis developed from gamma-irradiated seeds, to be used as an alternative method for producing L-DOPA. A \\
ultraperformance liquid chromatography system was used for L-DOPA quantification. The method was validated \\
and it revealed good sensitivity, specificity, precision, and accuracy. Irradiation of the seeds at a $200-500$ Gy gamma \\
dose exhibited the median lethal dose at 336.75 Gy. The shoots developed from seeds germinated after irradiation \\
with 200 and 300 Gy gamma dose produced L-DOPA at levels $300 \%$ and $220 \%$ higher than the nonirradiated group, \\
respectively, whereas the shoot formation was unsuccessful when the radiation increased to 400 and 500 Gy. The \\
results demonstrated that irradiation of the seeds at 200 and 300 Gy gamma can be used to produce in vitro shoots for \\
L-DOPA production.
\end{tabular}

\section{INTRODUCTION}

L-DOPA (known as levodopa and 3,4-dihydroxyphenylL-alanine) is a metabolite with diverse biological functions in humans and some plants. In humans, it is a precursor of dopamine which is a brain neurotransmitter and can penetrate the bloodbrain/blood-cerebrospinal fluid barrier. A deficiency of dopamine in the brain can result in motor function impairment, which is a major cause of physical disability in patients with Parkinson's disease (PD) (Jankovic, 2008). Since L-DOPA can increase the dopamine level in the brain, the administration of L-DOPA is commonly recommended for treating motor symptoms of PD (Nagatsu and Sawada, 2009). The current supply of L-DOPA for drug manufacturing is produced by the chemical synthetic process (Knowles, 2004). Nevertheless, the synthetic process for L-DOPA production is complex and produces low yield and low enantioselectivity (Min et al., 2015). Alternative approaches, such as biotransformation and microbial fermentation, have been

\section{"Corresponding Author}

Worapan Sitthithaworn, Faculty of Pharmacy, Srinakharinwirot University, Nakhonnayok, Thailand.E-mail:worapan@g.swu.ac.th utilized to improve the production yield and the enantioselectivity. Nevertheless, employment for industrial-scale production has been limited by low production capacity due to the complex purification process involved (Min et al., 2015).

It has been well established that Mucuna sp. (family Leguminosae) is rich in L-DOPA. Plant-derived L-DOPA is enantioselective, and the purification of L-DOPA from plants does not require a complicated process. It has been observed that Mucuna pruriens var. utilis contains 3.1\%-6.1\% L-DOPA in the seeds (Kavitha and Thangamani, 2014). The concentrations are high enough for commercial extraction. However, there are some limitations to producing L-DOPA from seeds. For instance, the pod has strident trichomes that cause a very strong itching sensation, and the success of cultivation greatly depends on the environmental conditions (Ingle, 2003). Therefore, in vitro plant cultivation has been an interesting alternative source for L-DOPA production because it takes a shorter time, and it is easier to control the environment during production. The formation of L-DOPA has been found in callus cultures (Brain, 1976), suspension cultures (Wichers et al., 1993), and regenerated plants (Lahiri et al., 2011) of M. pruriens var. utilis. However, the concentrations of L-DOPA were less than those accumulated in the seeds. 
Gamma rays are often employed to enhance the amounts of pharmacologically important metabolites. Gamma irradiation can be applied to plants or plant parts, as well as in vitro cultures (Vivek and Shukla, 2017). For example, irradiation of Psoralea corylifolia seeds at $20 \mathrm{kGy}$ resulted in an increase in the psoralen content as high as 32 -fold. The callus cultures of Stevia rebaudiana produced higher stevioside content after irradiation with 15 Gy. Irradiation of Panax ginseng hairy root cultures at $100 \mathrm{~Gy}$ stimulated saponin and ginsenosides contents as high as 1.4-fold and 1.8-fold, respectively. In addition, the camptothecin yield in the callus tissue of Nothapodytes foetida was also reported to increase by 20 -fold with $20 \mathrm{~Gy}$. Several studies have stated that gamma irradiation increased plant metabolite production by affecting the activity of secondary metabolite biosynthetic enzymes.

Gamma irradiation has also been applied to induce mutation in seeds of $M$. pruriens var. utilis for establishing plants producing a high level of L-DOPA(Singh et al., 2016). In the referenced study, gamma-ray irradiation at doses of 50-500 Gy was applied to the seeds and the mutant plants that provided high L-DOPA contents in the seed were selected. The mutant that produced the highest L-DOPA content could produce L-DOPA as twice as high as the parent plant. The study also revealed the suppression of the dopa-decarboxylase gene, which is responsible for the conversion of L-DOPA to dopamine, in the mutants with a high level of L-DOPA.

This research aimed to study the ability to produce L-DOPA in shoots of $M$. pruriens var. utilis cultivated in vitro from gamma-irradiated seeds, to be used as an alternative method for producing L-DOPA. In this study, a validated ultraperformance liquid chromatography (UPLC) method was used to determine the amount of L-DOPA in shoots (Wachisunthon et al., 2019).

\section{MATERIALS AND METHODS}

\section{Plant material}

The seeds of $M$. pruriens var. utilis Wall. ex Wight were obtained from plants grown in a greenhouse of the Medicinal Plant Research Institute, Department of Medical Sciences, Ministry of Public Health, Nonthaburi, Thailand.

\section{Gamma irradiation and shoot production}

Gamma irradiation was conducted at the Thailand Institute of Nuclear Technology at Naknonayok, Thailand, using a cobalt-60 source at a dosage rate of $115 \mathrm{~Gy} / \mathrm{hours}^{-1}$. Gamma irradiation was carried out at 0, 200, 300, and 400 Gy (30 seeds each). The total radiation dose absorbed by seeds was further confirmed by FWT50 alanine pellet dosimeters (Far West Technology, Inc., Goleta, CA). After irradiation, in vitro cultivated plants were prepared by adapting the method of Sathyanarayana et al. (2008). The seeds were surface-sterilized and germinated on Murashige and Skoog (MS) basal medium (Murashige and Skoog, 1962) at $25^{\circ} \mathrm{C} \pm 2{ }^{\circ} \mathrm{C}$ with a 16-hours light/8-hours dark photoperiod. On day 14, the number of sprouted seeds was recorded to determine the median lethal dose $\left(\mathrm{LD}_{50}\right)$. After germination, the nodal parts were used as explants and inoculated onto the MS medium supplemented with $2 \mu \mathrm{M}$ 6-benzylaminopurine (BAP). The cultures were incubated at $25^{\circ} \mathrm{C} \pm 2{ }^{\circ} \mathrm{C}$ under a 16 -hours photoperiod with a cool white fluorescent light for 30 days.

\section{Extraction of L-DOPA from in vitro cultivated plants}

The stems and the leaves of in vitro cultivated plants were dried using a hot air oven at $50^{\circ} \mathrm{C}$. The dried plants were ground to a fine powder with a granite mortar and pestle. The plants were extracted by adapting the method of Hasegawa et al. (2011). Ten milligrams of dried sample was mixed with $500 \mu \mathrm{l}$ of acetonitrile/ water/formic acid (50:50:1). The mixture was extracted using an ultrasound-assisted extraction method by sonication in an ultrasonic bath for 20 minutes at room temperature. Then, it was centrifuged at 10,000 rpm for 5 minutes. The supernatant was collected, and the precipitates were reextracted under the same conditions. After the second extraction, the precipitates were washed three times with $300 \mu \mathrm{l}$ of acetonitrile/water/formic acid $(50: 50: 1)$. All the supernatants were combined and produced up to $2 \mathrm{ml}$ using a volumetric flask.

\section{Sample preparation by solid-phase extraction (SPE)}

The extract was cleaned by SPE using a Sep-Pak si (500 mg; $3 \mathrm{ml} \mathrm{SPE} \mathrm{cartridge).} \mathrm{The} \mathrm{extract} \mathrm{was} \mathrm{wetted} \mathrm{with} 3$ $\mathrm{ml}$ of acetonitrile. Then, $100 \mu \mathrm{l}$ of the extract was loaded on the packing bed of the cartridge. Next, it was washed with $2 \mathrm{ml}$ of acetonitrile, and finally, L-DOPA was eluted from SPE with $4 \mathrm{ml}$ of methanol/water/formic acid ( $96: 3.5: 0.5)$. This final fraction was evaporated to a dry state using a nitrogen evaporator. The residue was dissolved with $200 \mu \mathrm{l}$ of acetonitrile/water/formic acid $(50: 50: 1)$ and filtered through a $0.2 \mu \mathrm{m}$ membrane filter before analysis by ultraperformance liquid chromatography (UPLC).

\section{Preparation of the standard solution}

A stock standard solution of $1 \mathrm{mg} \mathrm{ml}^{-1}$ was prepared by dissolving $2 \mathrm{mg}$ of standard L-DOPA (Sigma-Aldrich, St. Louis, MO) in $2 \mathrm{ml}$ of acetonitrile/water/formic acid (50:50:1). Working solutions with $5-100 \mu \mathrm{g} \mathrm{ml}^{-1}$ were prepared by diluting the stock solution with the same solvent.

\section{Chromatographic condition}

The analysis was carried out using an ultraperformance liquid chromatography (UPLC) system (Waters, Milford, MA) with a photodiode array detector. The chromatographic condition was developed and recently published elsewhere (Wachisunthon et al., 2019), i.e., the sample was separated on a Luna Omega Polar C18 $(150 \times 2.1 \mathrm{~mm}, 1.6 \mu \mathrm{m})$ column (Phenomenex, Milford, MA). The column temperature was $35^{\circ} \mathrm{C}$. The mobile phase was a mixture of $0.1 \%$ formic acid in water and methanol with a ratio of $94.6: 5.4$ at a flow rate of $0.25 \mathrm{ml} /$ minutes $^{-1}$. The injection volume was $1 \mu \mathrm{l}$, and the detection wavelength was $280 \mathrm{~nm}$. Method validation was carried out according to the ICH guidelines (ICH Expert Working Group, 2005).

\section{RESULTS AND DISCUSSION}

Gamma ray is an electromagnetic radiation that influences physiological and biochemical processes in plant cells and tissues, leading to the modification of plant phenotype and genotype (Sumira et al., 2012). These modifications rely on the intensity of radiation energy absorbed by plant tissues (Marcu et al., 2013). In this study, it is apparent that a high dose of gamma rays could hamper seed germination. Within a 14-days incubation period, the germination percentage observed from the 
nonirradiated seeds was $85 \%$. The germination rate diminished to $65 \%-75 \%$ when the seeds were treated with a 200-300 Gy gamma dose and decreased to less than $40 \%$ when they were treated with a 400-500 Gy gamma dose (Fig. 1A). The median $\mathrm{LD}_{50}$ determined based on the intersection of the $50 \%$ germination line with the germination curve was at 336.75 Gy (Fig. 1B). The appearance of the in vitro shoots is shown in Figure 2.

Gamma irradiation also affected subsequent shoot growth. The number of shoots was reduced in the groups exposed to the higher doses ( 400 and $500 \mathrm{~Gy}$ ) of gamma radiation compared to the control group (Table 1), and the shoots became deformed with yellow spots and were unable to survive. The results agreed with the radiation sensitivity testing reported previously, which showed that a low dose of gamma radiation was more effective for promoting seed germination and plant growth. For example, Lathyrus chrysanthus seed germination and shoot initiation were achieved by irradiation of seeds at 100-150 Gy gamma dose but were unsuccessful when the radiation increased to 200 Gy (Beyaz et al., 2016). It has also been reported for cucumber and okra seeds that greater numbers of seeds germinated after irradiation with a 50 Gy gamma dose compared to that with 100 and 200 Gy gamma doses (Norfadzrin et al., 2017). The ability of seed germination was diminished after exposure to higher doses of radiation, which may have been associated with chromosomal impairment and subsequent cell division retardation (Neelam et al., 2014).

This study also revealed that irradiation of $M$. pruriens var. utilis seeds with 200 and 300 Gy gamma doses could induce
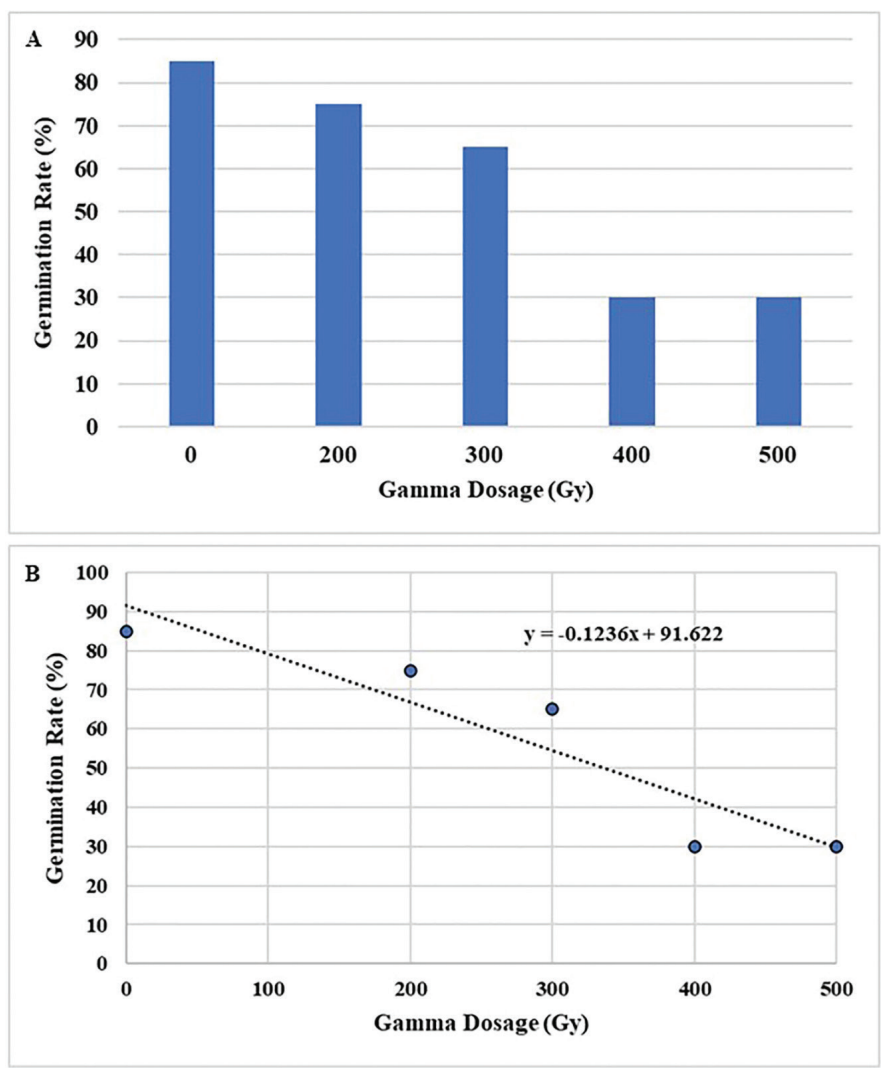

Figure 1. Germination percentages of gamma-irradiated seeds (A) and radiation survival curves (B) for M. pruriens var. utilis seeds. the accumulation of L-DOPA. The multiple shoots from the 200 and 300 Gy gamma doses were maintained for 30 days before they were harvested and measured for L-DOPA content and were found to contain L-DOPA at approximately two times the level of the nonirradiated group (Table 2 and Fig. 3). The yield of L-DOPA was greater for field-cultivated seeds $(3 \%-6 \%)$ than for in vitro shoots $(1.4 \%)$; however, the period from flowering to mature seed took 2-3 months, and the plants died 45-60 days after producing a seed (Fact Sheet - Mucuna pruriens - tropical Forages, 2018; Kavitha and Thangamani, 2014). Thus, the growing shoots in vitro were an appealing choice for L-DOPA production. In addition, the biomass amounts obtained from the 200 and 300 Gy gamma irradiation groups were 1.8 and 1.4 times higher than the nonirradiated group, respectively (Table 2). Taken together, considering the L-DOPA content and the biomass, shoots growing from seeds irradiated with 200 and 300 Gy gamma doses produced L-DOPA at levels of $300 \%$ and $220 \%$ higher than the nonirradiated group, respectively (Fig. 4).

The application of gamma radiation to enhance secondary metabolite accumulation or to induce synthesis has been supported by various authors (Ahamed, 2019; Akshatha et al., 2013; Fulzele et al., 2015; Kovacs and Keresztes, 2002). The gamma ray is known to be an abiotic elicitor that promotes the intracellular synthesis of oxygen radicals and hydrogen peroxide. These radicals may cause injury to plant cells and can affect certain important metabolic functions that might be crucial for plant survival (Wi et al., 2007). Production of L-DOPA might involve a defense system that responds to stress induced by gamma radiation.

In this study, L-DOPA was quantified using a UPLC method that was developed and validated for the separation and determination of L-DOPA in plantlets of M. pruriens var. utilis (Wachisunthon et al., 2019). Since plant leaves accumulate various metabolites that could impair the efficiency of analysis, SPE was applied to purify the sample before UPLC was performed. The method was suggested to be specific because the retention time (2.029 minutes) of the sample was equal to that of the L-DOPA standard, and there was no interference from the blank at the retention time of the standard. The calibration curve was linear

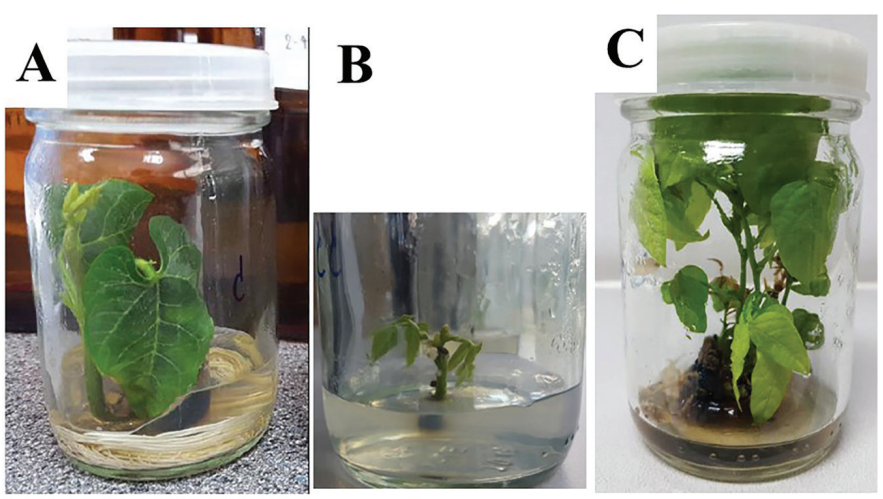

Figure 2. Mucuna pruriens var. utilis plantlets after seed germination (A), in vitro shoots initiated from a nodal explant cultured in the MS medium supplemented with $2 \mu \mathrm{M} \mathrm{BAP}$ at $25^{\circ} \mathrm{C} \pm 2{ }^{\circ} \mathrm{C}$ under a 16 -hours photoperiod with cool white fluorescent light (B), and in vitro shoots cultured for 30 days (C). The pictures are from the $200 \mathrm{~Gy}$ gamma dose. 
Table 1. Number of shoots and their appearance.

\begin{tabular}{ccl}
\hline Gamma radiation $(G y)$ & Number of shoots per explant (mean \pm SD) & Appearance \\
\hline 0 & $3 \pm 0.8$ & Green healthy leaves \\
200 & $3 \pm 1.5$ & Green healthy leaves \\
300 & $2 \pm 0.8$ & Green healthy leaves \\
400 & $0.8 \pm 0.3$ & The leaves were puckering and the colour was yellow. The plants die within 2 weeks. \\
500 & $1 \pm 0.5$ & The leaves were puckering and the colour was yellow. The plants die within 2 weeks. \\
\hline
\end{tabular}

Table 2. Biomass and L-DOPA content obtained from shoots after a 30-days incubation period.

\begin{tabular}{cccc}
\hline Radiation $(\mathrm{Gy})$ & Fresh weight $(\mathrm{g})$ & Dry weight $(\mathbf{m g})$ & L-dopa content* $(\boldsymbol{\%} \boldsymbol{w} / \boldsymbol{w})(\boldsymbol{\%} \mathbf{R S D}, \boldsymbol{n}=\mathbf{3})$ \\
\hline 0 & 1.81 & 175.23 & $0.67(1.38)$ \\
200 & 3.20 & 325.22 & $1.44(0.89)$ \\
300 & 2.45 & 252.55 & $1.49(1.58)$ \\
\hline
\end{tabular}
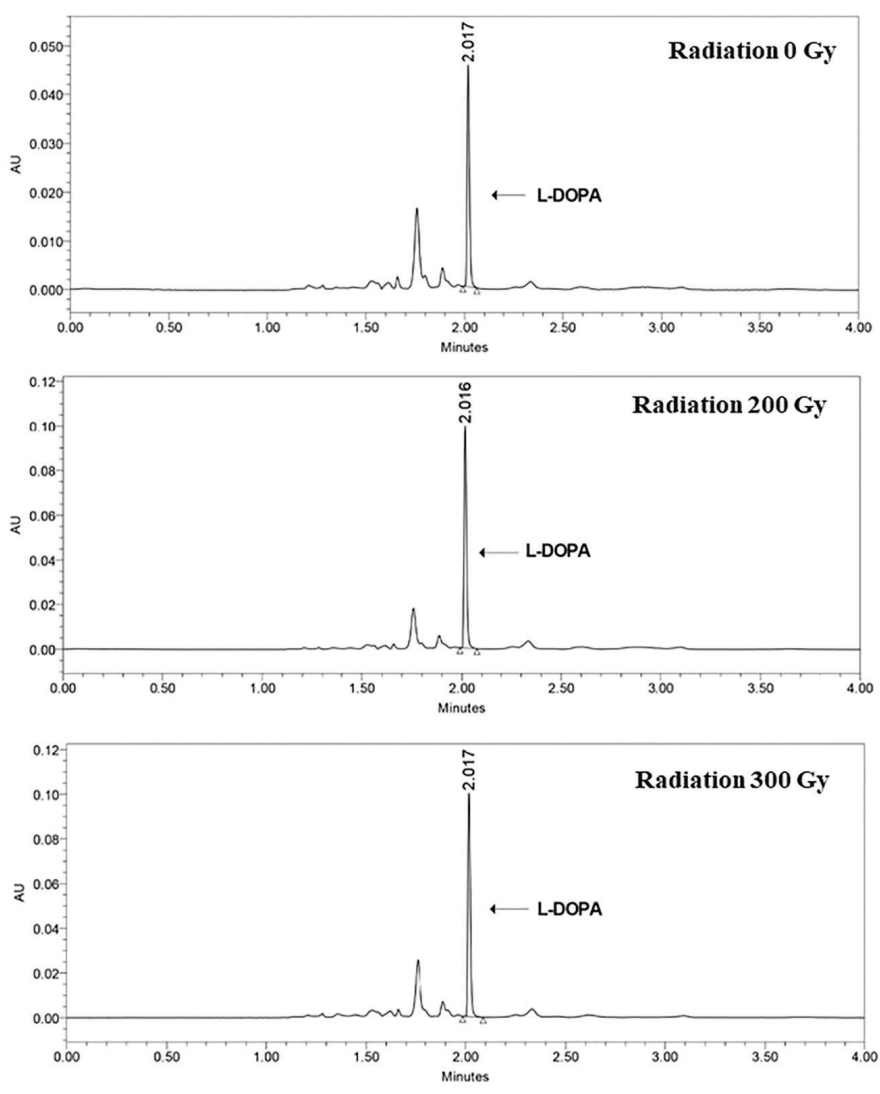

Figure 3. Chromatograms of L-DOPA derived from shoots after a 30-days incubation period.

at the concentration range of $5-125 \mu \mathrm{g} \mathrm{m}{ }^{-1}$ of L-DOPA, and the correlation coefficient $(\mathrm{R})$ was 0.9997 . The limit of detection and limit of quantitation were $3 \mu \mathrm{g} \mathrm{ml}^{-1}$ and $5 \mu \mathrm{g} \mathrm{ml}{ }^{-1}$, respectively. The precision studies revealed that the percent relative standard deviations ( $\%$ RSDs) of the L-DOPA content for repeatability and intermediate precision were 1.89 and 2.46 , respectively. Finally, the recovery of L-DOPA from spiked samples was $89 \%-92 \%$.

\section{CONCLUSION}

Acute gamma irradiation of $M$. pruriens var. utilis seeds at the doses of 200 and 300 Gy has been proven to enhance L-DOPA production from M. pruriens var. utilis shoot cultures.

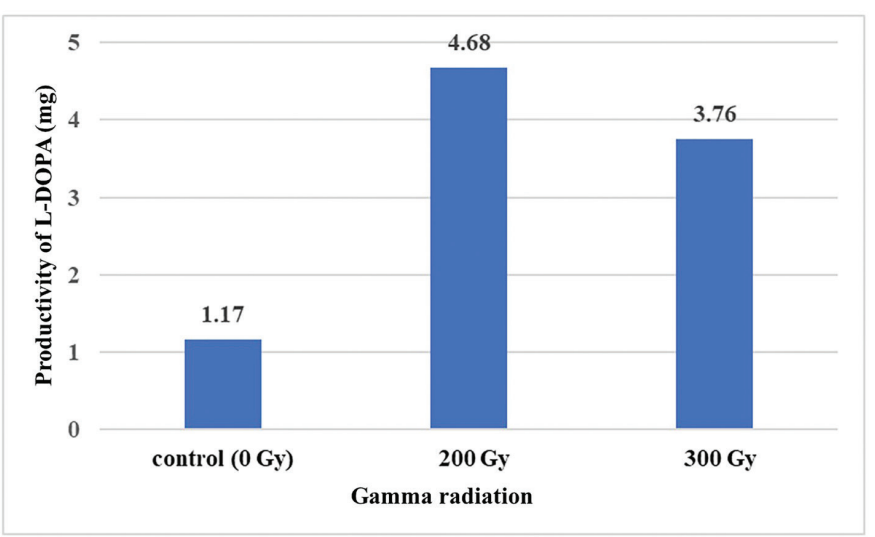

Figure 4. Productivity (calculated based on dried weight) of L-DOPA derived from shoots after a 30 -days incubation period.

However, further studies should be carried out to produce a highyielding clone, as well as maintain genetic stability, to establish genetically stable shoot cultures as an alternative L-DOPA source.

\section{ACKNOWLEDGMENTS}

This work was carried out with support from the Faculty of Pharmacy, Srinakharinwirot University. The authors are also thankful to the Medicinal Plant Research Institute, Department of Medical Science, Ministry of Public Health, and the Thailand Institute of Nuclear Technology (Public Organization) for providing the research facilities.

\section{AUTHOR CONTRIBUTIONS}

All authors made substantial contributions to conception and design, acquisition of data, or analysis and interpretation of data; took part in drafting the article or revising it critically for important intellectual content; agreed to submit to the current journal; gave final approval of the version to be published; and agree to be accountable for all aspects of the work.

\section{FUNDING}

There is no funding to report.

\section{CONFLICTS OF INTEREST}

The authors report no conflicts of interest in this work. 


\section{ETHICAL APPROVAL} subjects.

This study does not involve the use of animals or human

\section{REFERENCES}

Ahamed T. Bioprospecting elicitation with gamma irradiation combine with chitosan to enhance, yield production, bioactive secondary metabolites and antioxidant activity for saffron. J Plant Sci, 2019; 7(6):137-43.

Akshatha, Chandrashekar KR, Somashekarappa HM, Souframanien J. Effect of gamma irradiation on germination, growth, and biochemical parameters of Terminalia arjuna Roxb. Radiat Prot Environ, $2013 ; 36: 38-44$.

Beyaz R, Kahramanogullari C, Yildiz C, Darcin E, Yildiz M. The effect of gamma radiation on seed germination and seedling growth of Lathyrus chrysanthus boiss. Under in vitro conditions. J Environ Radioact, 2016; 162-163:129-33.

Brain KR. Accumulation of L-DOPA in cultures from Mucuna pruriens. Plant Sci Lett, 1976; 7(3):157-61.

Fact sheet - Mucuna pruriens - tropical Forages. Available via: http://www.tropicalforages.info/key/forages/Media/Html/entities/mucuna_ pruriens.htm (Accessed 20 September 2018).

Fulzele DP, Satdive R, Kamble S, Singh S, Singh S. Improvement of anticancer drug camptothecin production by gamma irradiation on callus cultures of Nothapodytes foetida. Int J Pharm Res Allied Sci, 2015; 4(1): $19-27$.

Hasegawa T, Ishii T, Takahashi K, Saijo M, Fukiwake T, Nagata T, Motoki Y. 2011. Quantitative determination of L-DOPA in dietary supplements containing Mucuna pruriens by high performance liquid chromatography. [ONLINE] Available via https//www.pref.chiba. lg.jp/eikn/eiseikenkyuu/shuppanbutsu/nenpou/documents/60tanpou2.pdf. (Accessed 02 October 2018).

ICH Expert Working Group. Validation of analytical procedures: text and methodology Q2(R1). In: Proceedings of the International Conference on Harmonisation of Technical Requirements for Registration of Pharmaceuticals for Human Use, Yogohama, Japan, 2005; pp 1-13.

Ingle PK. L-DOPA bearing plants. Indian J Nat Prod Resour, 2003; 2(3):126-33.

Jankovic J. Parkinson's disease. Clinical features and diagnosis. J Neurol Neurosurg Psychiatry, 2008; 79(4):368-76.

Kavitha C, Thangamani C. Amazing bean "Mucuna pruriens" a comprehensive review. J Med Plant Res, 2014; 8(2):138-43.

Knowles WS. 2004. Asymmetric hydrogenations-the monsanto L-DOPA process. In Blaser HU, Schmidt E. (ed.). Asymmetric catalysis on industrial scale. Weinheim, Germany: Wiley-VCH Verlag $\mathrm{GmbH} \&$ Co.KGaA 21-38.

Kovacs E, Keresztes A. Effect of gamma and UV-B/C radiation on plant cells. Micron. 2002; 33:199-210.

Lahiri K, Mukhopadhyay M, Mukhopadhyay S. Enhancement of L-DOPA production in micropropagated plants of two different varieties of Mucuna pruriens L., available in India. Plant Tissue Cult Biotechnol, 2011; 21(2):115-25.
Marcu D, Damain G, Cosma C, Cristea V. Gamma radiation effects on seed germination, growth and pigment content, and ESR study of induced free radicals in maize (Zea mays). J Biol Phys, 2013; 39:625.

Min K, Park K, Park D, Yoo Y. Overview on the biotechnological production of L-DOPA Appl Microbiol Biotechnol, 2015; 99:575-84.

Murashige T, Skoog F. A revised medium for rapid growth and bio assays with tobacco tissue cultures. Physiol Plant, 1962; 15(3):473-97.

Nagatsu T, Sawada M. L-dopa therapy for Parkinson's disease: past, present, and future. Parkinsonism Relat Disord, 2009; 15(Suppl 1): S3-8.

Neelam D, Tabasum T, Husain SA, Mahmooduzaffar S Radiation sensitivity of Cajanus Cajan to gamma radiations. J Food Process Technol, 2014; 5:394.

Norfadzrin F, Ahmed OH, Shaharudin S, Rahman DA. A preliminary study on radiosensitivity of tomato (Lycopersicon esculentum) and okra (Abelmoschus esculentus). Int J Agric Res, 2007; 2:620-5.

Sathyanarayana N, Kumar TB, Vikas PB, Rajesha R. In vitro clonal propagation of Mucuna pruriens var. utilis and its evaluation of genetic stability through RAPD markers. Afr J Biotechnol, 2008; 7(8): 973-80.

Singh S, Deepti Y, Lal RK, Gupta M, Dhawan S. Inducing Mutations through $\gamma$-irradiation in seeds of Mucuna pruriens for developing high L-DOPA-yielding genotypes. Int J Radiat Biol, 2016; 93:1-10.

Sumira J, Talat P, And T, Mahmooduzzafar N. Effect of gamma radiation on morphological, biochemical, and physiological aspects of plants and plant products. Environ Rev, 2012; 20:17-39.

Vivek PV, Shukla LI. Gamma irradiation of medicinally important plants and the enhancement of secondary metabolite production. Int J Radiat Biol, 2017; 93(9):967-79.

Wachisunthon D, Sitthithaworn W, Marsud S. 2019. Development and validation of an UPLC method for the determination of L-DOPA in plantlets of Mucuna pruriens var. utilis cultivated in vitro. The 35th International Annual Meeting in Pharmaceutical Sciences\& CU-MPU International Collaborative Research Conference. [ONLINE] Available via http://www.pharm.chula.ac.th/am2019/159-162. (Accessed 05 June 2020).

Wi SG, Chung BY, Kim JS. Effects of gamma irradiation on morphological changes and biological responses in plants. Micron, 2007; 38:553-64.

Wichers HJ, Visser JF, Huizing HJ, Pras N. Occurrence of L-DOPA and dopamine in plants and cell cultures of Mucuna pruriens and effects of 2,4-d and $\mathrm{NaCl}$ on these compounds. Plant Cell Tissue Organ Cult, 1993; 33:259.

How to cite this article:

Wachisunthon D, Marsud S, Poonsatha S, Jetawattana S, Sitthithaworn W. Productivity of L-DOPA in in vitro shoots of Mucuna pruriens var. utilis enhanced by gamma radiation. J Appl Pharm Sci, 2021; 11(01):084-088. 